\title{
Comparing Transform Domain Techniques and Vector Quantization Techniques for Face Detection and Recognition in Digital Images
}

\author{
T. K. Sarode \\ Phd,Thadomal Shahani College of Engg. \\ University of Mumbai, \\ Mumbai- 50,India.
}

\author{
Prachi Patil \\ Fr. Conceicao Rodrigues College of Engg. \\ University of Mumbai. \\ Mumbai- 50,India.
}

\begin{abstract}
Many face recognition algorithms have been proposed that help faces to be identified on systems. The aim of the paper is to compare the performance of transform domain techniques and Vector Quantization techniques. The system considers the full and partial feature vector sizes of images. In addition to this, the proposed system tries to improvise by extracting the face region before feature extraction is done. The system recognizes the face region using $\mathrm{YCbCr}$ color space to reduce the effect of lightening positions and intensities.
\end{abstract}

\section{General Terms}

Security, Biometric system.

\section{Keywords}

Face Detection, skin detection, transform domain techniques.

\section{INTRODUCTION}

Face Recognition and Detection system proposed here consists of three phases.

\section{Face Detection.}

In detection, the face region will be extracted from the input image using $\mathrm{YCbCr}$ color model.

2. Feature Extraction.

In second phase, feature vector will then be calculated using transform domain techniques like DCT, Walsh, Haar, Kekre's transforms and Vector Quantization (VQ) domain techniques like Linde-Buzo-Gray (LBG) Algorithm, Kekre's Proportionate Error Algorithm (KPE) and Kekre's Fast Codebook Generation (KFCG) Algorithm.

\section{Face Recognition.}

The feature vector will then be compared with the feature vectors for images stored in database. The image showing the minimum error will be the output of recognition process

This paper shows a comparison between recognition rates using different transform domain techniques and vector quantization techniques.

Figure 1 shows the steps involved in the proposed system.

The remainder of this paper is organized as follows: in section 2 the face detection procedure is discussed followed by section 3 which focuses on face recognition procedure. Results are elaborated in section 4 and conclusion has been outlined in section 5 .

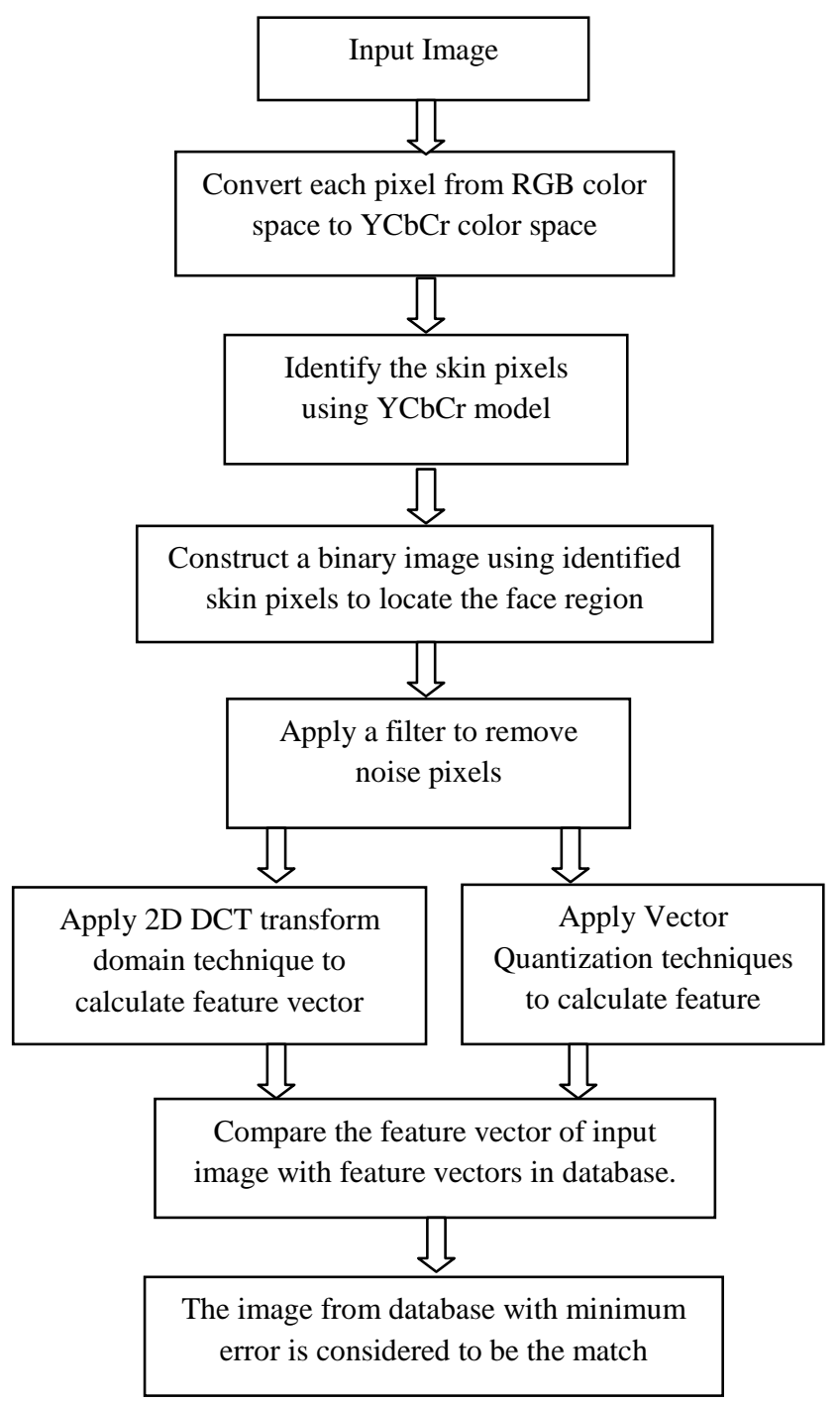

Figure 1. Shows the block diagram of proposed system.

\section{FACE DETECTION}

Face detection tries to identify the face regions in the entire image using $\mathrm{YCbCr}$ color space[1]. The $\mathrm{Cb}$ and $\mathrm{Cr}$ components help to identify skin regions in input image based on thresholds. The result of this step is a binary image which identifies the position of different skin pixels. 
The pixels are marked as skin pixels which fall in the range of set thresholds. The threshold values for the range of $\mathrm{CB}$ and $\mathrm{Cr}$ are identified using experimental results.

$\mathrm{r} 1<\mathrm{Cr}(\mathrm{i}, \mathrm{j})<\mathrm{r} 2$

$\mathrm{r} 3<(\mathrm{Cb}(\mathrm{i}, \mathrm{j})+0.6 * \mathrm{Cr}(\mathrm{i}, \mathrm{j}))<\mathrm{r} 4$

$\mathrm{r} 5<\mathrm{Cb}(\mathrm{i}, \mathrm{j})<\mathrm{r} 6$

where $\mathrm{i}, \mathrm{j}$ are current pixel position and $1<\mathrm{i}, \mathrm{j}<\mathrm{N}, \mathrm{N}$ is number of pixels in rows and columns. $\mathrm{r} 1$ to $\mathrm{r} 5$ are positive integer values which vary for different skin tones.

Figure 2 shows a sample of input image and its equivalent binary image constructed using $\mathrm{Cb}$ and $\mathrm{Cr}$ components.
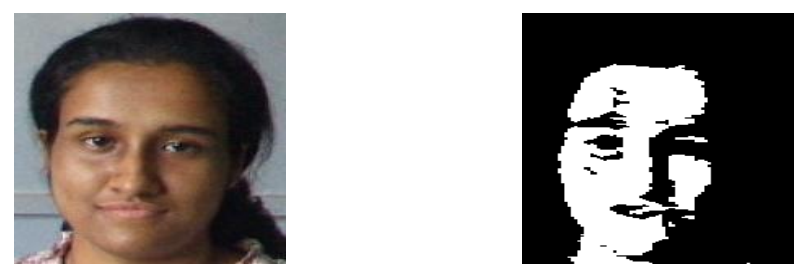

Figure 2. Shows the sample input image and result of skin region detection.
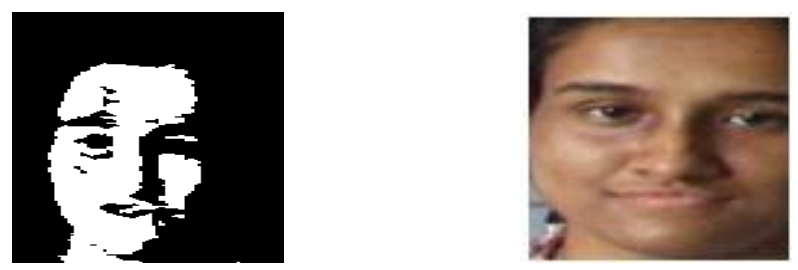

Figure 3. Shows the face region extracted from binary image.

Figure 3 shows binary image and extracted skin area from entire image.

The result of this stage is given as an input to face recognition process.

\section{FACE RECOGNITION}

Once the face region has been identified, the next step is to correctly identify the face. To match among different faces detected, feature vectors are calculated for each image using transform domain techniques and Vector Quantization techniques[16].

The feature vector of input image is them compared with feature vectors of other images in database. The image with minimum error value is considered to be the correct match.

The error value is calculated using mean squared error metric.

$\mathrm{MSE}=\frac{\sum_{\mathrm{M}, \mathrm{N}}\left[\mathrm{I}_{1}(\mathrm{~m}, \mathrm{n})-\mathrm{I}_{2}(\mathrm{~m}, \mathrm{n})\right]^{2}}{\mathrm{M} * \mathrm{~N}}$

Where $\mathbf{M}$ and $\mathbf{N}$ are the number of rows and columns respectively in the input images.

The steps for feature extraction are as follows:
1. Calculate the feature vector using various transforms on extracted face. Store the features of input image and various other images in database.

2. Calculate the mean square error to find the difference between feature vectors of extracted face from input image and images in database. If both the features vectors matches, then face is correctly identified.

The following subsection discusses the different techniques applied to calculate feature vector.

\subsection{Feature Vector using Transform Domain Techniques.}

2D Transform domain techniques like DCT[2][3], Walsh[4], Haar and Kekre's[5] are applied over the output of face detection phase[7].

Feature vectors are even calculated by using row mean and column mean[8]. The row mean vector is the set of averages of the intensity values of the respective rows. The column mean vector is the set of averages of the intensity values of the respective columns

Row Mean Vector =

[Avg(Row1), Avg(Row2),..... Avg(Row n)]

Column Mean Vector $=$

$[\operatorname{Avg}(\operatorname{Col~1)}), \operatorname{Avg}(\operatorname{Col} 2), \ldots \ldots \ldots \operatorname{Avg}(\operatorname{Col} n)]$

A new feature vector is calculated by arranging the row mean vector in first column and column mean vector in second column in the resultant vector.

Row-Column Mean Vector $=$

[Row_mean_Vector Column_Mean_Vector]

\subsection{Feature Vector using Vector Quantization Techniques.}

Vector Quantization (VQ) is an efficient technique for data compression and is very popular in a variety of research fields such as image segmentation, speech data compression, content based image retrieval CBIR and face recognition. Various techniques like Linde-Buzo-Gray (LBG)[9][10][11] Algorithm, Kekre's Proportionate Error Algorithm (KPE) [12] and Kekre's Fast Codebook Generation (KFCG)[13][14][15] Algorithm of VQ are used.

Feature vector of varying sizes are calculated by considering the partial co-efficients[6] from top left corner of 2D coefficients matrix and different algorithms are compared based on these feature vectors.

\section{RESULTS}

Table 1 shows the results of 2D transforms applied over Indian Face Database of 1000 test images. 
Table 1. Shows accuracy $(\%)$ for different feature vector sizes when 2D transforms are applied to extracted faces.

\begin{tabular}{|c|c|c|c|c|}
\hline \multirow{2}{*}{} & \multicolumn{4}{|c|}{ Transform Domain Techniques 2D } \\
\cline { 2 - 5 } & DCT & WALSH & HAAR & KEKRE \\
\hline $\mathbf{4} * \mathbf{4}$ & 83.16 & 15.3 & 15.3 & 15.3 \\
\hline $\mathbf{8} * \mathbf{8}$ & 79.08 & 21.93 & 21.93 & 21.93 \\
\hline $\mathbf{1 6} * \mathbf{1 6}$ & 78.57 & 32.14 & 32.14 & 22.95 \\
\hline $\mathbf{3 2} * \mathbf{3 2}$ & 78.06 & 42.34 & 42.34 & 32.14 \\
\hline $\mathbf{6 4} * \mathbf{6 4}$ & 78.06 & 55.61 & 55.61 & 42.85 \\
\hline $\mathbf{1 2 8} * \mathbf{1 2 8}$ & 78.06 & 68.87 & 68.87 & 47.74 \\
\hline $\mathbf{2 5 6} * \mathbf{2 5 6}$ & 77.55 & 77.55 & 77.55 & 37.75 \\
\hline
\end{tabular}

It can be seen that the recognition rate increases as the feature vector sizes increases whereas the recognition rate in DCT increases when feature vector size is minimum. The highest recognition rate of $83.16 \%$ for feature vector size $4 * 4$ is achieved using DCT transform amongst all other techniques. $77.55 \%$ accuracy is achieved using Haar and Walsh transform for feature vector size $256 * 256$ and $47.74 \%$ accuracy is achieved for Kekre transform using vector size $128 * 128$.

Results of applying 1D transforms (Row Mean) technique are tabulated below in table 2 .

Table 2. Shows accuracy (\%) for different feature vector sizes for 1D transforms applied on row mean of extracted faces.

\begin{tabular}{|c|c|c|c|c|}
\hline \multirow{2}{*}{} & \multicolumn{4}{|c|}{$\begin{array}{c}\text { Transform Domain Techniques 1D } \\
\text { (Row Mean) }\end{array}$} \\
\cline { 2 - 5 } & DCT & WALSH & HAAR & KEKRE \\
\hline $\mathbf{4} * \mathbf{1}$ & 43.87 & 6.63 & 37.75 & 18.36 \\
\hline $\mathbf{8} * \mathbf{1}$ & 56.63 & 8.16 & 53.06 & 19.89 \\
\hline $\mathbf{1 6} * \mathbf{1}$ & 58.16 & 14.28 & 57.14 & 22.95 \\
\hline $\mathbf{3 2} * \mathbf{1}$ & 56.63 & 16.83 & 57.14 & 30.1 \\
\hline $\mathbf{6 4} * \mathbf{1}$ & 56.63 & 27.04 & 56.63 & 42.34 \\
\hline $\mathbf{1 2 8} * \mathbf{1}$ & 56.63 & 37.24 & 56.63 & 57.14 \\
\hline $\mathbf{2 5 6} * \mathbf{1}$ & 56.63 & 56.63 & 56.63 & 56.63 \\
\hline
\end{tabular}

It is observed that the recognition rate for DCT is $58.16 \%$ using feature vector size of $16^{*} 1$ is highest among all techniques. The recognition rates for other techniques increases with increase in feature vector sizes. For Walsh and Haar technique the highest recognition of $56.63 \%$ each is achieved for feature vector size of $256 * 1$ and Kekre transform shows a result of $57.14 \%$ at feature vector size $128 * 1$.

Table 3 represents results of applying 1D column mean technique over the images.
Table 3. Shows accuracy ( $\%$ ) for different feature vector sizes for 1D transforms applied on column mean of extracted faces.

\begin{tabular}{|c|c|c|c|c|}
\hline \multirow{2}{*}{} & \multicolumn{4}{|c|}{$\begin{array}{c}\text { Transform Domain Techniques 1D } \\
\text { (Column Mean) }\end{array}$} \\
\cline { 2 - 5 } & DCT & WALSH & HAAR & KEKRE \\
\hline $\mathbf{1} * \mathbf{4}$ & 45.6 & 6.63 & 44.89 & 21.93 \\
\hline $\mathbf{1 * 8}$ & 56.12 & 10.2 & 55.61 & 24.48 \\
\hline $\mathbf{1} * \mathbf{1 6}$ & 58.16 & 11.22 & 57.14 & 27.04 \\
\hline $\mathbf{1} * \mathbf{3 2}$ & 59.18 & 18.87 & 58.16 & 34.18 \\
\hline $\mathbf{1} * \mathbf{6 4}$ & 58.67 & 28.57 & 58.67 & 45.91 \\
\hline $\mathbf{1} * \mathbf{1 2 8}$ & 58.16 & 41.32 & 58.67 & 52.55 \\
\hline $\mathbf{1} * \mathbf{2 5 6}$ & 58.67 & 58.67 & 58.67 & 58.67 \\
\hline
\end{tabular}

It is observed that DCT shows the highest accuracy of $59.18 \%$ for vector size $1 * 32$ followed by Walsh, Haar and Kekre transforms with accuracy of $58.67 \%$ for vector size $1 * 256$.

Results of applying 1D transform (Row-Column Mean technique) are shown below in Table 4.

Table 4. Shows accuracy (\%) for different feature vector sizes for 1D transforms applied on row-column mean of extracted faces.

\begin{tabular}{|c|c|c|c|c|}
\hline & \multicolumn{5}{|c|}{$\begin{array}{c}\text { Transform Domain Techniques 1D } \\
\text { (Row-Column Mean) }\end{array}$} \\
\cline { 2 - 5 } & DCT & WALSH & HAAR & KEKRE \\
\hline $\mathbf{4} * \mathbf{2}$ & 64.79 & 9.69 & 60.71 & 28.57 \\
\hline $\mathbf{8} * \mathbf{2}$ & 74.48 & $11 . .73$ & 73.97 & 32.14 \\
\hline $\mathbf{1 6} * \mathbf{2}$ & 75.51 & 17.34 & 75.51 & 43.36 \\
\hline $\mathbf{3 2} * \mathbf{2}$ & 75.51 & 25 & 76.02 & 48.46 \\
\hline $\mathbf{6 4} * \mathbf{2}$ & 75 & 39.79 & 75.51 & 55.61 \\
\hline $\mathbf{1 2 8} * \mathbf{2}$ & 75 & 49.48 & 75 & 68.87 \\
\hline $\mathbf{2 5 6} * \mathbf{2}$ & 75 & 75 & 75 & 75 \\
\hline
\end{tabular}

From the results it is observed that Haar transform gives maximum accuracy of $76.02 \%$ for feature vector size $32 * 2$. DCT has a maximum recognition rate of $75.51 \%$ for vector size 16*2 followed by Walsh and Haar transform with accuracy of $75 \%$ for vector size $256 * 2$.

Table 5 shows the result of applying vector quantization techniques.

Table 5 Accuracy (\%) for different feature vector sizes for VQ techniques applied on extracted faces.

\begin{tabular}{|c|c|c|c|}
\hline \multirow{2}{*}{} & \multicolumn{3}{|c|}{ VQ Domain Techniques } \\
\cline { 2 - 4 } & LBG & KPE & KFCG \\
\hline $\mathbf{4}^{* 12}$ & 10.2 & 10.2 & 43.87 \\
\hline $\mathbf{8} * \mathbf{1 2}$ & 11.22 & 12.24 & 48.97 \\
\hline $\mathbf{1 6} * \mathbf{1 2}$ & 13.77 & 13.26 & 52.04 \\
\hline $\mathbf{3 2} * \mathbf{1 2}$ & 10.71 & 11.22 & 54.59 \\
\hline $\mathbf{6 4} * \mathbf{1 2}$ & 10.71 & 13.26 & 60.71 \\
\hline $\mathbf{1 2 8} * \mathbf{1 2}$ & 14.79 & 15.81 & 67.34 \\
\hline $\mathbf{2 5 6} * \mathbf{1 2}$ & 21.93 & 18.36 & 63.77 \\
\hline
\end{tabular}


It is observed from the different VQ technique, that KFCG techniques results maximum accuracy rate of $67.34 \%$ for feature vector size of $128 * 12$. The recognition rates of LBG and KPE are considerably poor with accuracy of $21.93 \%$ and $18.36 \%$ respectively.

\section{CONCLUSION}

In this paper face recognition using transform domain and vector quantization technique is compared. Transform domain techniques like DCT, Walsh, Haar and Kekre's are used. LBG, KPE and KFCG techniques of VQ domain are considered. The proposed system compares the full and partial coefficients of images. Different feature vector sizes are calculated by considering coefficients from top left corner of images.

After applying different transform domain techniques, the recognition rate is maximum when feature vectors are calculated using 2D-DCT transform. The performance is maximum with $83.16 \%$ accuracy rate for minimum feature vector size of $4 * 4$ in case of DCT transform. Whereas the Walsh, Haar and Kekre transform results are maximum when feature vector size is maximum. The application of row mean(maximum of $58.16 \%$ ) and column mean (maximum of $59.18 \%$ ) techniques does not out-perform in comparison to $2 \mathrm{D}$ transforms. Whereas the recognition rates after combining row mean and column mean (maximum of $76.02 \%$ ) are good in comparison to applying row mean and column mean individually.

The highest recognition rate in both the result set is $83.16 \%$ using 2D DCT transform with the smallest feature vector size of $4 * 4$, giving up the advantage.

Among the applied techniques of Vector Quantization, KFCG has shown better performance than LBG and KPE technique. The recognition rate of $67 \%$ is the maximum for vector size of $128 * 2$ using KFCG technique. Whereas LBG and KPE techniques have failed to give performance.

\section{REFERENCES}

[1] Mohamed Berbar, Hamdy Kelash, "Faces and Facial Features Detection in Color Images", GMAI'06, pp. 1-6, 2006.

[2] Andrew B. Watson, "Image compression using the Discrete Cosine Transform”, Mathematica journal, 4(1), pp. 81-88, 1994

[3] H.B.Kekre, Tanuja Sarode, Sudeep D. Thepade, "DCT Applied to Row Mean and Column Vectors in Fingerprint Identification", In Proceedings of International Conference on Computer Networks and Security (ICCNS), 27-28 Sept. 2008, VIT, Pune.

[4] Dr. H. B. Kekre, Sudeep Thepade, Archana Athawale, Anant Shah, Prathamesh Verlekar, Suraj Shirke. "Walsh Transform over Row Mean and Column Mean using Image Fragmentation and Energy Compaction for Image Retrieval". / (IJCSE) International Journal on Computer Science and Engineering, Vol. 02, No.01S, 2010, 47-54.

[5] "Image Retrieval using Non-Involutional Orthogonal Kekre's Transform", International Journal of Multidisciplinary Research and Advances in Engineering (IJMRAE), Ascent Publication House, 2009, Volume 1, No.I, pp 189-203, 2009.
[6] H.B.Kekre, Sudeep D. Thepade, "Improving the Performance of Image Retrieval using Partial Coefficients of Transformed Image", International Journal of Information Retrieval (IJIR), Serials Publications, Volume 2, Issue 1, 2009, pp. 72-79.

[7] H. B. Kekre, S. Thepade, A. Maloo. "Performance Comparison of Image Retrieval Using Fractional Coefficients of Transformed Image Using DCT, Walsh, Haar and Kekre's Transform”. CSC-International Journal of Image processing (IJIP), 4(2)142-155, 2010.

[8] H.B.Kekre, S. D. Thepade, A. Athawale, A. Shah, P. Verlekar, S. Shirke. "Performance Evaluation of Image Retrieval using Energy Compaction and Image Tiling over DCT Row Mean and DCT Column Mean". Springer-International Conference on Contours of Computing Technology (Thinkquest-2010), Babasaheb Gawde Institute of Technology, Mumbai, 2010.

[9] H.B.Kekre, K. Shah, T. K. Sarode, S. Thepade. "Performance Comparison of Vector Quantization Technique-KFCG with LBG, Existing Orthogonal Transforms and PCA For Face Recognition". International Journal of Information Retrieval, II(I):6471, 2009.

[10] Y. Linde, A. Buzo, R. M. Gray. "An algorithm for vector quantizer design". IEEE Transaction on Communication, COM-28(1):84-95, 1980.

[11] A. Gersho, R.M. Gray. "Vector Quantization and Signal Compression", Kluwer Academic Publishers, Boston, 1991.

[12] H. B. Kekre, T. Sarode. "Two Level Vector Quantization Method for Codebook Generation using Kekre's Proportionate Error Algorithm". CSC-International Journal of Image Processing, 4(1):1-10, 2010.

[13] H.B. Kekre, T. Sarode.” An Efficient Fast Algorithm to Generate Codebook for Vector Quantization". First International conference on Emerging Trends In Engineering and Technology (ICETET), 2008.

[14] H.B.Kekre, T. K. Sarode, S. D. Thepade, V. Suryavanshi. "Improved Texture Feature Based Image Retrieval using Kekre's Fast Codebook Generation Algorithm". Springer-International Conference on Contours of Computing Technology (Thinkquest-2010), Babasaheb Gawde Institute of Technology, Mumbai, 2010.

[15] H. B. Kekre, Ms. T. K. Sarode, S. D. Thepade. "Image Retrieval using Color-Texture Features from DCT on VQ Codevectors obtained by Kekre's Fast Codebook Generation". ICGST- International Journal on Graphics, Vision and Image Processing (GVIP), 9(5):1-8, 2009.

[16] Dr. H. B. Kekre, Dr. Tanuja K. Sarode, Prachi J. Natu , Shachi J. Natu. "Performance Comparison of Face Recognition Using DCT Against Face Recognition Using Vector Quantization Algorithms LBG, KPE, KMCG, KFCG" International Journal Of Image Processing (IJIP), Volume (4),377-389. 